Revue de droit comparé du travail et de la sécurité sociale

3 | 2018

La participation des travailleurs dans la grande entreprise privée et publique

\title{
Les droits de participation des travailleurs
}

Michel Coutu, Gilles Auzero et Isabelle Daugareilh

\section{(2) OpenEdition \\ Journals}

Édition électronique

URL : https://journals.openedition.org/rdctss/1877

DOI : $10.4000 /$ rdctss. 1877

ISSN : 2262-9815

Éditeur

Centre de droit comparé du travail et de la sécurité sociale

Édition imprimée

Date de publication : 1 novembre 2018

Pagination : 6-13

ISSN : 2117-4350

\section{Référence électronique}

Michel Coutu, Gilles Auzero et Isabelle Daugareilh, « Les droits de participation des travailleurs », Revue de droit comparé du travail et de la sécurité sociale [En ligne], 3 | 2018, mis en ligne le 01 novembre 2021, consulté le 13 novembre 2021. URL : http://journals.openedition.org/rdctss/1877 ; DOI : https:// doi.org/10.4000/rdctss. 1877

\section{(c) (i) $\odot$}

Revue de droit comparé du travail et de la sécurité sociale est mise à disposition selon les termes de la Licence Creative Commons Attribution - Pas d'Utilisation Commerciale - Pas de Modification 4.0 International. 


\title{
LES DROITS DE PARTICIPATION DES TRAVAILLEURS ${ }^{1}$
}

\author{
Michel COUTU \\ Professeur, Université de Montréal, CRIMT
}

GiLles AuZERO

Professeur, COMPTRASEC, Université de Bordeaux

\section{ISABELLE DAUGAREILH \\ Directeur de Recherche, CNRS, COMPTRASEC, Université de Bordeaux}
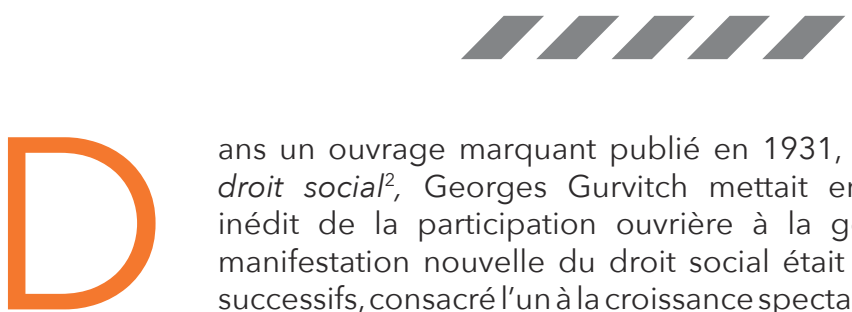

ans un ouvrage marquant publié en 1931, Le temps présent et l'idée de droit social2, Georges Gurvitch mettait en lumière le développement inédit de la participation ouvrière à la gestion des entreprises. Cette manifestation nouvelle du droit social était abordée dans deux chapitres successifs, consacré l'un à la croissance spectaculaire des accords collectifs de travail, l'autre à l'émergence du « contrôle ouvrier » proprement dit. S'inspirant en particulier de la doctrine allemande, au premier chef des travaux de Hugo Sinzheimer ${ }^{3}$, Gurvitch soulignait que la nouvelle « constitution du travail » (Arbeitsverfassung) ${ }^{4}$, limitant fortement l'absolutisme hier incontesté des droits de direction du patron, revêtait un caractère de

1 Les articles publiés dans ce dossier sont issus d'un séminaire international organisé conjointement par le CRIMT (Centre de recherche interuniversitaire sur la mondialisation et le travail - Canada) et le COMPTRASEC (Centre d'étude comparée du droit du travail et de la sécurité sociale) et qui s'est tenu à Bordeaux les 22 et 23 septembre 2017. Ce séminaire s'inscrivait dans le cadre du projet international de recherche Better Institutions for Better Work Partnership Project initié par le CRIMT avec le soutien du Conseil de recherches en sciences humaines du Canada et dont le COMPTRASEC est partie prenante : il bénéficia de l'appui des universités de Paris-Dauphine, Brême, Montréal et Louvain-La-Neuve (UCL). Nous souhaitons adresser des remerciements pour les échanges scientifiques que nous avons eus à cette occasion avec Gregor Murray et Philippe Barré (Université de Montréal), Blanche Segrestin (MINES Paris Tech), Thierry Kirat (Université ParisDauphine), Martine Le Friant (Université d'Avignon) et Marie-Ange Moreau (COMPTRASEC). Nous signalons également la contribution à l'organisation du séminaire et aux discussions apportée par des collègues présents par visioconférence (en simultané) à l'Université de Montréal, à savoir Nicolas Roby, Isabelle Martin et Francine Jacques

2 G. Gurvitch, Le temps présent et l'idée de droit social, Paris, Éditions du Recueil Sirey, Paris, 1931, sp.pp. 13-99.

3 H. Sinzheimer, Grundzüge des Arbeitsrechts, 2e ed., Jena, Gustav Fischer, 1927, sp. pp. 207 et s.

4 À notre connaissance, Max Weber fut l'un des premiers auteurs de langue allemande à faire un usage extensif du concept de " constitution du travail » dans ses travaux de jeunesse, tout à fait remarquables, consacrés au sort des travailleurs agricoles dans l'Est de l'Allemagne. Voir M. Weber, «Entwickelungstendenzen in der Lage der ostelbischen Landarbeiter»,1894, dans les Gesammelte Aufsätze zur Sozial- und Wirtschaftsgeschichte, Tübingen, J.C.B. Mohr, 1988, pp. 470-507. Déjà chez Weber le concept présente une tournure bien davantage sociologique que juridique, alors que c'est l'inverse qui prévaudra lorsque le concept sera repris par la doctrine travailliste. 
droit autonome, d'ordre institutionnel plutôt que contractuel, et axé vers la démocratisation de l'entreprise. Pour Gurvitch, ce détachement de la gestion de l'entreprise du droit de propriété résultait soit de l'institution de mécanismes obligatoires de participation des travailleurs (les conseils d'entreprise, Gurvitch donne ici l'exemple de l'Allemagne, de l'Autriche, etc.), soit, d'une manière plus indirecte, de l'enserrement des droits de direction dans la régulation dense découlant des conventions collectives (Angleterre, États-Unis).

Gurvitch, se référant en cela à des auteurs français, autrichiens et allemands, et avant tout à Sinzheimer, partait d'une conception institutionnaliste de l'entreprise, celle-ci constituant dans le cadre de l'économie capitaliste un ordre juridique autonome, en fait une structure de domination soumise ab initio à l'absolutisme du patron. Pour contrer l'arbitraire patronal et favoriser la prise en considération des intérêts opposés des travailleurs, diverses formes de participation des salariés ont été imaginées, visant l'actionnariat, l'organisation et la gestion du travail, enfin la présence de représentants au sein même des organes directeurs de l'entreprise. Pour Gurvitch, certaines de ces formules sont illusoires ou trompeuses: ainsi, la participation au capital qui risque de lier l'ouvrier-actionnaire aux aléas économiques de l'entreprise ; de même, l'institution par l'employeur même de comités dits représentatifs qui érigent autant d'obstacles à la présence syndicale dans l'établissement. Par contre, alors que l'impact de la codécision en Allemagne reste à mesurer, l'institution de conseils d'entreprise, garantie par l'article 165 de la Constitution de Weimar, disposition dont Sinzheimer fut le rédacteur, montre la voie possible vers une certaine démocratie industrielle, fruit de l'autodétermination sociale, nullement en contradiction avec l'action syndicale et ne constituant pas davantage une récupération du droit social autonome par l'État : au contraire, par sa nécessaire intervention, ce dernier favorise l'autonome collective, sans l'inféoder de quelque manière à la volonté étatique.

Plusieurs décennies plus tard, alors qu'entretemps la participation des travailleurs connut d'abord une éclipse complète sous les totalitarismes, puis un renouveau significatif après la Seconde guerre mondiale, en Allemagne d'abord, puis dans plusieurs pays européens, sans que les formes de la codécision et de la cogestion ne soient aucunement reçues en Amérique du Nord, en Angleterre et dans les autres pays anglo-saxons, Otto Kahn-Freund, influencé lui-aussi par Sinzheimer dont il fut l'étudiant, s'interrogea sur l'état de la démocratie industrielle en Grande-Bretagne ${ }^{5}$. L'occasion lui en fut fournie par le Rapport Bullock (1977) portant sur cette question et qui recommandait l'adoption par le Parlement britannique d'une loi imposant aux grandes entreprises l'ouverture de leurs conseils d'administration à des représentants des travailleurs. Kahn-Freund se montra hostile à l'idée, ce qui contribua sûrement à la mise au rencard du rapport. Sur le plan structurel, il estimait qu'en l'absence de l'infrastructure indispensable qui consiste en l'existence préalable d'influents conseils d'entreprise, comme c'est le cas en Allemagne, cette introduction par le haut d'un zeste de démocratie industrielle ne mènerait nulle part. Mais, plus fondamentalement, Kahn-Freund se demandait quelle était la logique de tout ce branle-bas, alors que la forte présence des syndicats britanniques au niveau de l'établissement imposait des contraintes très significatives aux employeurs, au moins équivalentes sinon supérieures à celles existant en Allemagne.

Indépendamment de ce qu'il advint par la suite (l'échec du « laissez-faire collectif » dans sa résistance aux contre-réformes qu'allait introduire le gouvernement Thatcher), il faut retenir de

5 O. Kahn-Freund, "Industrial Democracy", Industrial Law Journal, 1977, Vol. 6, n 1, pp. 65-84. 
cette lecture comparée de Gurvitch et de Otto Kahn-Freund que les voies de la participation des travailleurs sont multiples, parfois surtout symboliques, fréquemment au contraire empiriquement effectives. En outre, il faut replacer ces phénomènes dans leur cadre plus général, en tenant compte de l'aménagement de la participation des travailleurs (au sens large) dans les systèmes de relations professionnelles en cause, suivant notamment qu'ils prévoient une représentation essentiellement moniste (la négociation collective conduite par les syndicats représentatifs) ou dualiste (syndicats, conseils d'entreprise/cogestion) des travailleurs.

Si I'on aborde la question de la participation des travailleurs dans une perspective de philosophie sociale et du travail comme nous y invite Dominique Méda en brossant un vaste tableau d des réflexions qui ont prévalu dès la seconde moitié du XIXème siècle en France sur l'auto-organisation des ouvriers, on est frappé par le caractère très actuel des questions alors posées. Les premières réflexions visaient à surmonter la condition ouvrière au moyen de l'association des producteurs, sous la forme de la coopérative ou de la propriété fédéraliste (Proudhon). Au tournant du siècle, le régime associationniste est défendu par Jean Jaurés, mais aussi par Adéodat Boissard, un juriste français spécialisé en droit du travail: ce dernier s'élève contre ce qu'il dénomme le «participationnisme», qui concerne la pseudo participation des ouvriers aux bénéfices de l'entreprise. La logique de cette forme d'association au capital voudrait que les salariés assument aussi des tâches de direction et d'autorité, mais ceci leur est bien entendu refusé. Pire encore, dépendants de décisions économiques et financières sur lesquelles ils n'ont aucun contrôle, les ouvriers deviennent parties prenantes des risques encourus par le patron et dont ils peuvent faire les frais.

Si l'idée de «participation-association » a semblé un temps mise de côté compte tenu des progrès de la négociation collective et du droit du travail, elle a fait périodiquement l'objet de plaidoyers soutenus. Ainsi, en 1985, le politologue Robert Dahl développait à son tour une critique appuyée de l'autoritarisme régnant dans l'économie, en particulier en contexte étatsunien, et argumentait en faveur d'une démocratisation de l'entreprise. Il invoquait notamment à cet égard un droit moral à la participation aux décisions économiques, tout en restant vague cependant sur les modalités concrètes d'une telle participation.

Si l'on quitte précisément le domaine des théories de la justice économique pour s'interroger sur ses modalités, au moins quatre avenues peuvent être identifiées : la gestion participative dans l'entreprise par l'introduction d'équipes semi-autonomes, -ce qui n'y modifie en rien la structure des pouvoirs; la formation de coopératives comme alternatives au modèle entrepreneurial dominant, -encore que dans les faits celles-ci se conforment largement au style de gouvernance de l'entreprise capitaliste; la cogestion et la codétermination à l'allemande, modèle qui s'est répandu peu ou prou dans l'ensemble de l'Europe; enfin, la participation directe des travailleurs au gouvernement de l'entreprise, ce qu'lsabelle Ferreras qualifie de bicaméralisme.

Prenant le relai sous l'angle de la sociologie des relations professionnelles et en adoptant une posture critique influencée, entre autres, par les travaux de Jürgen Habermas, Isabelle Ferreras revisite la célèbre analyse tridimensionnelle de la citoyenneté chez T.H. Marshall, rattachant les apports du droit du travail au versant social de celle-ci. Pour Ferreras, les droits fondamentaux du travail, loin d'être des manifestations secondaires ou dérivées de la citoyenneté sociale, devraient plutôt être associés à la sphère des droits politiques. Seule cette manière d'envisager les droits de participation des travailleurs permet de lier adéquatement économie et politique dans une vision critique des démocraties contemporaines, contre la perspective du libéralisme cherchant à isoler les deux sphères l'une de l'autre. S'est ainsi développée une contradiction manifeste entre démocratie 
et capitalisme, entre aspirations des citoyens à la participation dans l'espace public et domination de plus en plus extensive de la vie sociale par les acteurs économiques. Or, cette contradiction se vit de manière aiguë au sein de l'entreprise, où les salariés apparaissent " tiraillés entre le monopole du pouvoir concédé aux seuls apporteurs en capital dans leur vie de travail et l'aspiration à l'égalité démocratique ». Ce constat oblige à repenser la structuration des pouvoirs dans l'entreprise, dans une perspective de démocratisation. À cet égard, Ferreras en appelle à l'institutionnalisation, au-delà des comités d'entreprise et (en Allemagne) de la codétermination, d'un espace de gouvernement représentant les intérêts des travailleurs dans l'entreprise, au moyen de ce qu'elle appelle le bicaméralisme.

La participation des travailleurs dans l'entreprise n'est pas qu'une idée politique et sociale. C'est aussi un droit qui a été consacré dans de nombreux systèmes juridiques, parfois dans les textes fondamentaux et en tout cas de manière diverse selon les systèmes voire au sein de chaque système. Le choix des pays retenus pour illustrer les modes de participation des travailleurs permet d'une part d'aborder de manière critique les modèles (Allemagne, Norvège, Suède) et d'autre part de présenter un nuancier des formules juridiques retenues (Pays Bas, France, Canada, Belgique), tout en prenant en considération des systèmes qui ne sont pas allés au-delà d'aspirations politiques (Belgique) comme de ceux qui poursuivent sans y parvenir complètement cet objectif (France). Wolfgang Däubler rappelle l'origine des conseils d'établissement en Allemagne, apparus dans la foulée du mouvement des conseils lors de la Révolution de Novembre 1918, auxquels la République de Weimar devait reconnaître un fondement constitutionnel. En 1947, les puissances d'occupation favorisèrent la réintroduction de la cogestion, avec l'appui du patronat lequel redoutait la socialisation des entreprises. Pour saisir la cogestion dans sa dynamique actuelle, il faut tenir compte du modèle dualiste de gouvernance des grandes sociétés (par actions ou à responsabilité limitée) prévalant en Allemagne : au directoire chargé de l'administration des affaires courantes se surajoute le conseil de surveillance, lequel contrôle le premier et en valide certaines décisions. Seul le conseil de surveillance est concerné par la cogestion.

Celle-ci implique trois cas de figures : $1^{\circ}$ dans les sociétés comptant entre 500 et 2000 salariés, le tiers des membres du conseil de surveillance sont élus par les travailleurs ; 2 ' lorsque la société emploie plus de 2000 travailleurs, ceux-ci élisent la moitié des membres du conseil de surveillance, à ceci près que le président, lequel détient une voix prépondérante, est en pratique toujours un représentant du capital ; $3^{\circ}$ enfin, dans le secteur du charbon et de l'acier, la parité entre capital et travail est pleinement réalisée au conseil de surveillance : les représentants respectifs des salariés et des actionnaires choisissent de concert le président du conseil et élisent les membres du directoire. Par arrangement tacite, seul le « directeur du travail » agit à titre de représentant des travailleurs.

Dans tous les cas, la marge de manœuvre des représentants des travailleurs est cependant limitée par l'obligation de loyauté envers l'entreprise, laquelle implique notamment le respect de la confidentialité. En outre, remarque Däubler, le côté travail se trouve souvent désavantagé par le manque d'information et d'expertise quant aux dossiers complexes qui sont discutés au conseil de surveillance. Le problème peut s'avérer particulièrement aigu en cas de restructurations de l'entreprise : à cet égard, la négociation collective, validée sur ce thème par les tribunaux du travail, offre ici des perspectives plus prometteuses que la cogestion.

Dans une contribution distincte, Christoph Teichmann et Justin Monsenepwo apportent des précisions complémentaires. La cogestion forme avec la négociation collective l'un des 
deux piliers du partenariat social allemand. La philosophie sociale en repose sur le principe d'équilibre et d'égalité entre les droits du travail et ceux du capital : elle entend donc compenser, au profit des travailleurs, la prépondérance de la volonté patronale qui subsiste en dépit des conventions collectives et de la législation du travail. II importe toutefois de bien différencier la cogestion au sein de l'établissement - la structure réelle de travail - et qui repose sur le conseil d'établissement, et celle qui s'exerce au sein du conseil de surveillance, laquelle dépend des formes juridiques de la société qui chapeaute l'entreprise. Un conseil d'établissement doit être mis sur pied dès que le lieu de travail compte au moins cinq salariés : la cogestion s'étend alors aux décisions portant sur les conditions de travail, telles les heures de travail, le temps supplémentaire ou les méthodes de travail. En conséquence, l'employeur ne peut prendre de décisions en matière de gestion collective du travail sans obtenir au préalable l'accord formel du conseil d'établissement.

En ce qui concerne la participation des travailleurs à la gestion de la société, les auteurs apportent diverses précisions relatives à la stricte séparation entre gestion et surveillance en droit allemand des sociétés. Cette séparation implique en particulier la limitation du rôle du conseil de surveillance à sa seule fonction de contrôle et l'impossibilité d'obtenir simultanément un mandat au sein du directoire et du conseil de surveillance. À tout événement, soulignent Teichmann et Monsenepwo, les acteurs prépondérants demeurent ici les actionnaires, ce qui est conforme à la garantie constitutionnelle du droit de propriété découlant de la Loi fondamentale. Tout en admettant que la cogestion assure une concertation sociale permanente au sommet de l'entreprise et fonde sa légitimité, les auteurs relèvent le fréquent contournement des règles par la création de filiales européennes ou étrangères, et soulèvent la question d'une réforme de la cogestion allemande.

Présentant le modèle nordique de dialogue social, Bernard Johann Mulder en met en exergue l'élément essentiel, lequel se fonde sur la confiance, entre les membres et la direction des syndicats, mais aussi entre les partenaires sociaux eux-mêmes, enfin entre ceux-ci et les gouvernements et autorités administratives. Comme partout, le droit du travail s'est érigé ici dans le sillage des troubles sociaux, mais l'acceptation de certaines prérogatives des employeurs et la reconnaissance mutuelle des associations syndicales et patronales a permis la coopération. En outre, l'État a laissé une grande latitude aux partenaires sociaux dans l'autorégulation des relations de travail, reconnaissant la conciliation et l'arbitrage, et accordant une pleine valeur juridique aux conventions collectives, favorisant ainsi un niveau élevé de syndicalisation. Pour Mulder, le modèle social nordique se caractérise par les éléments suivants : le haut niveau d'organisation à la fois des salariés et des employeurs ; l'importance des conventions collectives, lesquelles déterminent les salaires et les autres conditions de travail ; enfin, le rôle limité de la loi, comparé à la position qu'elle assume dans la plupart des pays européens. Concernant cet aspect, l'auteur insiste sur l'influence considérable qu'exercent les partenaires sociaux sur le contenu de la législation. Mais cette dimension passe nettement au second plan, comparée à l'importance fondamentale de l'autorégulation, au point que l'auteur évoque, en transposant les propos de Otto Khan-Freund, une version nordique du « laissez-faire collectif » axé sur la négociation collective.

La notion de démocratie au travail, soutient Mulder, implique le droit pour les salariés et les syndicats d'exercer une influence significative sur les décisions de l'employeur. Tant en Norvège qu'en Suède, la participation des travailleurs aux conseils de surveillance des entreprises est garantie par la loi.

Jan Cremers indique d'emblée qu'aux Pays-Bas, la présence syndicale directe au niveau de l'entreprise et de l'établissement demeure modeste, sauf chez certains grands employeurs. 
C'est pourquoi, les comités d'entreprise, imposés par voie législative, sont devenus le canal majeur de la représentation syndicale au niveau local. La loi prévoit l'obligation pour toute entreprise comptant 50 salariés de mettre sur pied un comité d'entreprise, lequel est doté de droits à l'information, de consultation et de codécision. Notons que les droits de consultation concernent notamment la question des restructurations : en cas de désaccord, le comité d'entreprise possède un droit d'appel devant la Cour d'appel, laquelle peut dans certains cas bloquer la décision. Quant à la codécision, elle porte sur les retraites, le temps de travail, le salaire et les barèmes de la rémunération, la politique de réintégration, le recrutement et les autres politiques relatives au personnel.

La législation prévoit également une représentation, indirecte toutefois, des travailleurs au sein du conseil de surveillance des sociétés comptant au moins cent salariés. La société néerlandaise obéit en effet à une structure duale de gouvernement, soit, comme en Allemagne, le directoire et le conseil de surveillance. Le comité d'entreprise joue ici un rôle pivot, puisqu'il peut dans certains cas élire le tiers des membres du conseil de surveillance, lesquels ne peuvent cependant être des salariés. En pratique, l'expérience se révèle mitigée : les syndicats et les salariés n'accordent qu'une importance très secondaire à ces droits de participation, nullement équivalents à ce qui existe en Allemagne, et favorisent plutôt un renforcement du pouvoir des comités d'entreprise.

Examinant successivement le droit du travail et le droit des sociétés en Belgique, Filip Dorssemont et Auriane Lamine mettent en lumière le refus du constituant belge, en dépit de la consécration de droits économiques, sociaux et culturels en 1993, d'affirmer un droit des travailleurs à la participation à la gestion, à la différence des acquis historiques des constitutions françaises, italiennes ou allemandes. Le constituant s'est limité à garantir uniquement des droits de consultation et d'information, ne s'avançant guère sur la voie d'une démocratisation de l'entreprise. À cet égard, les auteurs distinguent la codétermination de la cogestion. Alors que la première fait obstacle à l'adoption unilatérale de décision ou de règles par le chef de l'entreprise, la seconde concerne le pouvoir de gérer celle-ci au quotidien.

En droit belge, toute entreprise employant au moins 50 salariés est tenue d'instituer un conseil d'entreprise. La loi institue en outre des comités pour la prévention et la protection au travail (CPPT) et une délégation syndicale (DS) : seule cette dernière n'est pas une instance mixte. Ces instances interviennent dans l'exercice par la direction de ses pouvoirs règlementaire, disciplinaire et de gestion. Ainsi, le conseil d'entreprise, et non plus le chef d'entreprise, a seul compétence pour élaborer et modifier le règlement d'entreprise. Par contre, le pouvoir disciplinaire reste l'apanage exclusif de l'employeur, tout en étant assujetti au règlement d'entreprise. Enfin, le pouvoir de gestion est le corollaire de la liberté d'entreprise et des droits de propriété des employeurs. II en résulte que les projets de restructurations ne peuvent être contestés par les travailleurs, sauf du point de vue de leurs conséquences sociales. En matière de gestion, la compétence du conseil d'entreprise se limite en particulier aux questions touchant l'organisation du travail et la gestion des œuvres sociales instituées par l'entreprise en vue du bien-être de ses salariés. Par ailleurs, en droit belge, les interactions entre le droit du travail et le droit des sociétés demeurent limitées. Seul le rôle du réviseur d'entreprise apparaît ici significatif ; en outre, le droit des sociétés n'impose pas de représentation des travailleurs au sein de l'organe d'administration de la société. Bien que la revendication d'une démocratie économique s'inscrive au cœur des demandes des syndicats belges depuis près d'un siècle, il n'y a pas eu de consensus sur les moyens d'atteindre cet objectif. 
Outre Atlantique, au Québec précisément, Julie Bourgault et Michel Coutu estiment qu'il y aurait peu à dire à ce sujet si l'on ne prenait en considération que l'impact de la négociation collective. En effet, le droit québécois du travail ignore la représentation dualiste (syndicats et comités d'entreprise) qui caractérise la plupart des systèmes européens de relations industrielles. Comme le veut le modèle Wagner, c'est essentiellement par un système fortement décentralisé de négociation collective, avec reconnaissance par accréditation étatique du seul syndicat majoritaire au regard d'une unité de négociation, que se réalise la participation des travailleurs à l'organisation des relations de travail dans l'entreprise. Certes, la législation du travail oblige parfois l'entreprise à se doter d'instances représentatives du personnel, y compris dans le secteur non syndiqué: par exemple, en matière de santé et de sécurité du travail ou de reclassement des salariés. Toutefois, en l'absence d'une représentation syndicale dans l'entreprise, ${ }^{6}$ de tels comités semblent dépourvus d'une réelle efficacité. Par ailleurs, il est rarissime que des représentants des travailleurs puissent siéger au conseil d'administration des entreprises, même si le Code du travail envisage sommairement cette possibilité.

En ce qui concerne les milieux syndiqués, les auteurs relèvent les nombreuses faiblesses que présente le système actuel de négociation collective au Québec, à commencer par l'absence d'accès à la syndicalisation des cadres intermédiaires et subalternes, et des soidisant "entrepreneurs », en fait des employés économiquement dépendants. De même, l'obligation législative de paix industrielle et le principe de l'appui majoritaire au syndicat comme précondition sine qua non de la syndicalisation représentent autant d'obstacles au plein épanouissement de la négociation collective. En outre, Bourgault et Coutu mettent en relief la collision fréquente entre la négociation collective, et la liberté d'entreprendre et le droit de propriété, normes fondamentales (encore que non-écrites) de la « constitution économique » au Canada. Ainsi, la fermeture antisyndicale du magasin Wal-Mart de Jonquière au Québec a semblé consacrer la primauté absolue de ces normes économiques sur les droits collectifs des travailleurs. Toutefois, au terme d'un combat épique devant les tribunaux lequel s'étendit sur une décennie, les 190 salariés du Wal-Mart de Jonquière ont finalement eu gain de cause, touchant des indemnités compensatoires substantielles. Cette victoire en bout de piste devant la Cour suprême du Canada serait peu compréhensible sans l'arrière-plan du revirement jurisprudentiel opéré par la Cour, à partir de 2007, en matière de liberté syndicale : un mouvement de constitutionnalisation du droit du travail a été enclenché à partir de ce moment, dont les contours précis demeurent encore indéfinis, mais qui, se réclamant d'emblée d'une pleine conformité aux normes internationales du travail, contient en germe la possibilité d'une transformation en profondeur du système québécois des relations industrielles et des droits de représentation et de participation des travailleurs qui en découlent.

En France, la participation des travailleurs à la gestion des entreprises repose sur un fondement constitutionnel, soit l'alinéa 8 du Préambule de la Constitution de 1946 ainsi que le rappelle Sylvain Niquège. Qu'en est-il de ce principe dans les grandes entreprises nationales relevant du secteur public de l'économie? Le secteur public ne se réfère pas à cet égard à une forme juridique spécifique d'entreprise, pouvant par exemple s'étendre à des sociétés d'économie mixte. Quoiqu'il en soit, la question essentielle consiste à se demander si le secteur public se montre davantage favorable que le secteur privé à la participation des travailleurs. Niquège observe qu'il n'existe pas de modèle participatif qui serait propre aux grandes entreprises du secteur public. Au regard du code du travail, l'entreprise, qu'elle soit

6 Au Québec, le taux de syndicalisation demeure élevé par rapport au reste du Canada et aux ÉtatsUnis, puisqu'il atteint $40 \%$ de la main-d'œuvre. Ce taux est stable depuis plusieurs décennies. 
publique ou privée, obéit aux mêmes exigences en matière de participation, ce qui n'exclut pas toutefois certains aménagements spécifiques.

Il importe également de tenir compte de l'absence d'homogénéité de statut des personnels de l'entreprise publique. Ce phénomène d'hybridation se traduit par la présence simultanée de fonctionnaires, d'agents publics et d'agents de droit privé. Par exemple, chez France Télécom/Orange et à La Poste, la transformation de l'entité publique en société commerciale a résulté en une telle hybridation des statuts des salariés. À La Poste, le législateur a choisi de ne pas introduire les modalités de participation prévues au Code du travail, tout en autorisant l'élection par l'ensemble des travailleurs, indépendamment du statut, des membres des comités techniques et du comité d'hygiène, de sécurité et des conditions de travail. Par contre, chez France Télécom/Orange, c'est l'option contraire qui fut retenue, soit l'application du droit commun de la représentation.

S'agissant du secteur privé de l'économie en France, Gilles Auzero distingue deux dispositifs de participation à la gestion : la cogestion concerne les règles assurant la représentation des salariés au sein des organes de direction de la société employeur ; la codécision suppose que certaines décisions ne peuvent être prises sans le consentement des représentants du personnel. Jusqu'à récemment, le droit français ne s'intéressait guère à l'une ou l'autre de ces formes de la participation. Le comité d'entreprise, qui est désormais désigné " comité social et économique ${ }^{7}{ }^{7}$ ne dispose pour l'essentiel que d'attributions consultatives, sauf en matière d'horaires individualisés, de nomination du médecin du travail et du reclassement interne de salariés visés par un licenciement pour motif économique.

Cela dit, certains changements sont à l'œuvre dans le système juridique français. En matière de codécision, le Code du travail prévoit qu'en vertu d'un accord collectif d'entreprise ou de branche, le comité économique et social pourra se transformer en un "conseil d'entreprise " doté de prérogatives plus étendues, notamment en matière de formation. Ils ont d'abord concerné, en 2013, la cogestion. Toutefois, met en garde Auzero, « ... au total le développement de la codécision dans notre droit positif est abandonné à la volonté des partenaires sociaux, c'est-à-dire à un accord de l'employeur et des syndicats de salariés. II est loin d'être certain que le premier accepte ainsi librement de se déposséder d'une partie de son pouvoir de décision. »

En matière de cogestion cette fois, la Loi relative à la sécurisation de l'emploi du 14 juin 2013 impose la présence de représentants des salariés dotés d'une voix délibérative au sein des conseils d'administration et de surveillance. Ce dispositif est limité aux sociétés, filiales comprises, qui emploient au moins mille salariés. Par ailleurs, le Code du travail prévoit que le comité d'entreprise peut déléguer deux de ses membres pour participer aux séances du conseil d'administration ou de surveillance, avec voix consultative. L'auteur relève toutefois les nombreuses insuffisances du droit français en la matière : ainsi, les sociétés à responsabilité limitée (SARL) et les sociétés par actions simplifiée (SAS), de plus en plus nombreuses, ne sont pas visées par l'obligation de représentation des salariés. Par ailleurs, la financiarisation de l'économie contribue à favoriser la gouvernance de la société par les actionnaires: or, les salariés n'ont pas accès à l'assemblée générale des actionnaires, sauf indirectement (délégués du comité d'entreprise) et en ne pouvant s'exprimer que très marginalement.

7 Le comité social et économique résultera de la fusion des délégués du personnel, du comité d'entreprise et du comité d'hygiène, de sécurité et des conditions de travail. 